\title{
A role for Gen5 in heterochromatin structure, gene silencing and NER at the $H M L$ locus in budding yeast
}

\author{
Hua Chen ${ }^{1}$, Ling Zhang, Qikai Wang, Chenxi He, Lauren Frances Dender, Feng Gong ${ }^{1}$ \\ Department of Biochemistry and Molecular Biology, University of Miami Miller School of \\ Medicine, Miami, Florida 33101
}

Running title: Gcn5 functions on heterochromatic $H M L$

${ }^{1}$ To whom correspondence should be addressed:

Dr. Feng Gong

Department of Biochemistry and Molecular Biology

University of Miami Miller School of Medicine, Miami, Florida 33136

Email: fgong@med.miami.edu

Dr. Hua Chen

Department of Biochemistry and Molecular Biology

University of Miami Miller School of Medicine, Miami, Florida 33136

Email: hchen2@med.miami.edu 


\begin{abstract}
Histone acetyltransferase Gcn5 plays an important role in transcription activation, DNA replication-coupled nucleosome assembly and nucleotide excision repair (NER). However, its functions on the heterochromatin are unexplored. Here, we find that removal of Gcn5 leads to more condensed heterochromatin structure, as revealed by topology analysis of $H M L$ circles. Importantly, the altered heterochromatin structure is restored by re-expression of Gen5 in the gcn $5 \Delta$ cells. As a result of the more compact heterochromatin, gene silencing at the $H M L$ locus is increased and NER efficiency at $H M L$ is impaired in the absence of Gcn5. Interestingly, while the association of SIR complex with $H M L$ is enhanced in cells lacking Gcn5, the altered compaction of $H M L$ heterochromatin is also observed due to the deletion of Gen5 from Sir'cells. These findings reveal a role of Gcn5 in the regulation of heterochromatin structure, gene silencing and NER efficiency at the heterochromatic $H M L$ locus in yeast.
\end{abstract}

Key words: Gcn5, heterochromatin, $H M L$, SIR complex, NER, Silencing 


\section{Introduction}

Genome in eukaryotes is compacted to chromatin. The degree of chromatin compaction is not homogenous across the genome, leading to the existence of highly condensed regions known as heterochromatin or silenced chromatin and less condensed regions referred to as euchromatin $(1,2)$. While gene expression is largely inactive, heterochromatin plays important roles in gene regulation and genome stability (3). Alerted heterochromatic state can impair gene expression patterns, leading to the development of different human diseases (4). The transcriptionally silent $H M$ loci including $H M L$ and $H M R$ located on chromosome $\amalg$ are a well-known heterochromatin locus in budding yeast Saccharomyces cerevisiae. Formation and maintenance of silenced HM loci require the silent information regulator (SIR) complex composed of Sir2, Sir3 and Sir4 (5). In the absence of SIR complex, HML heterochromatin is fully derepressed (6). On the other hand, overexpression of SIR complex is toxic to yeast cells by causing chromosome loss (7). In addition to the SIR complex, several other proteins have been identified to be involved in formation and/or maintenance of heterochromatin structure of $H M L$, such as the chromatin remodeler Fun30 (6) and the NER (Nucleotide Excision Repair) protein Rad4 (8).

Gen5 (General Control Non-depressible 5) is one of the best characterized histone acetyltransferase (HAT), belonging to the GNAT (Gcn5-related $N$-acetyltransferases) family of acetyltransferase enzymes (9). It functions in context of the transcriptional activator complex SAGA (10). Gcn5 acetylates histones at multiple positions including H3K9 and H3K14 (11-13), while recent studies indicated it can also acetylate many non-histone substrates $(14,15)$. Moreover, acetyltransferase activity of Gcn5 to acetylate histones is weak on its own and the optimal activity requires Ada2 and Ada3 $(16,17)$, another two subunits of SAGA complex. Ada2 
physically interacts with Gcn5 and promotes its activity by enhancing binding of the enzymatic cosubstrate acetyl-CoA (18).

Gcn5 plays important roles in replication-coupled nucleosome assembly and DNA repair $(19,20)$. It is generally considered to function on euchromatin because of the nature of being an acetyltransferase that activates transcription by acetylating nucleosomal histones (21). Currently its roles on heterochromatin are unclear. However, it has been reported that Ada2, the coactivator of Gen5, is involved in transcriptional silencing at telomeres and ribosomal DNA (22). Furthermore, Gcn5-mediated histone acetylation is critical to efficient NER at a repressed yeast locus (11). These studies suggest a correlation between Gcn5 and the repressed or silenced chromatin. Here, we investigated the function of Gen5 on $H M L$ heterochromatin structure. It was found that Gen5 is involved in the compaction of $H M L$. Removal of Gen5 resulted in more condensed $H M L$ as revealed by topological analysis of $H M L$ circles. In addition, the heterochromatin in gcn5 $\Delta$ cells is further silenced and NER of UV damage is impaired. These data identified Gcn5 as one more factor that contributes to the heterochromatin structure of $H M L$ and established a novel role of Gen5 in budding yeast. 


\section{Results}

\section{Altered heterochromatin structure at the $H M L$ locus in the gcn $5 \Delta$ cells}

Chromosome structure can be reflected by the topology of DNA. To examine the structure of heterochromatic $H M L$ locus, we analyzed the DNA topology of $H M L$ using a method established in previous work (23). In this method, two recombination target sites $(F R T)$ for the site-specific recombinase Flp1 were inserted in direct orientation at positions flanking $H M L$ (Fig. 1A, YXB2) or bracketing the coding region of $H M L$ and excluding the silencers (Fig 1A, YXB4). Galactose induction of Flp1 from a GAL10-FLP1 construct resident elsewhere on chromosome leads to recombination between the two $F R T$ sites and subsequent excision of $H M L$ as non-replicating mini-chromosome circles which are negatively supercoiled $(8,23)$. Topoisomers of these $H M L$ circles can be separated by chloroquine-agarose gel electrophoresis. In the presence of $30 \mu \mathrm{g} / \mathrm{ml}$ chloroquine, the more negatively supercoiled circles migrate more slowly in the gel which can be visualized by Southern blotting (23). The topology of $H M L$ between different strains can be compared using the difference of linking number $(\Delta \mathrm{Lk})$.

Inspired by our earlier work revealing that NER protein Rad4 regulates the heterochromatin conformation of $H M L$ and telomere (8), we set out to investigate whether other Rad proteins in the $\operatorname{Rad} 3$ epistasis group (24) including $\operatorname{Rad} 1, \operatorname{Rad} 7, \operatorname{Rad} 14$, and $\operatorname{Rad} 23$ are involved in the regulation of $H M L$ structure. Results of Southern blots (Fig. S1) indicated that removal of Rad1, Rad7 and Rad 14 didn't affect the DNA topology of $H M L$ circles. In contrast, deletion of $\operatorname{Rad} 23$ led to more negatively supercoiled $H M L$ circles, similar to that observed in Rad4A cells (8). Given Rad23 interacts with Rad4 forming the Rad4-Rad23 heterodimer which is crucial for NER (25), we assume the Rad4-Rad23 heterodimer plays a role in heterochromatin structure at the $H M L$ locus. 
Because it has been known that histone modifications (26-28) and histone chaperones (29-31) play a critical role in heterochromatin silencing in yeast, we next examined the involvement of two histone acetyltransferases (Gcn5 ,Rtt109), and two chromatin assembly factors (Asf1, Caf1) in the regulation of heterochromatin structure at $H M L$ locus. As shown in Fig. $1 \mathrm{~B}$ and $1 \mathrm{~F}$, topologies of $H M L$ circles in YXB4 (WT) and the gcn5s strain were different with $\Delta$ Lk of $\sim 1$. Moreover, those circles isolated from the gcn5s cells were more negatively supercoiled, suggesting $H M L$ was more compacted in the gcn5s cells than WT. On the other hand, less topology changes were observed when the RTT109 acetyltransferase was disrupted (Fig.1, C and G). Additionally, topologies of $H M L$ circles were not altered by removal of ASF1 and CAF1

(Fig. 1, D, E, H and I), suggesting these two histone chaperones are not involved in building up the heterochromatin structure of $H M L$ although they have been proven to contribute to the silencing of $H M L$ (29-31). Subsequent studies focused on Gcn5 which contributed most to $H M L$ structure among these four tested factors.

\section{Re-expression of Gen5 in the gcn $5 \Delta$ cells restores $H M L$ heterochromatin structure}

To test if $H M L$ heterochromatin structure in $g c n 5 \Delta$ cell can be restored by re-expression of Gcn5, a low copy $C E N$ vector carrying the GCN5 gene under the control of its native promoter was introduced into wild type (YXB4) and the gcn5s cells. Topology analyses by Southern blotting (Fig. 2, A and B) indicated that the existence of empty vector in WT cells didn't affect the topologies of $H M L$ circles (Fig. 2A, lane 2). In addition, overexpression of GCN5 in WT cells didn't alert the $H M L$ topology either (Fig. 2A, lane 1), suggesting cellular Gen5 is abundant for maintaining the heterochromatin structure of $H M L$. Importantly, re-expression of GCN5 in gcn5 5 cells led to similar supercoiling of $H M L$ circles to that in wild type (Fig. 2A, lanes 2 and 
4), restoring the altered heterochromatin structure of $H M L$ resulted from deleting GCN5 (Fig. 2A, lane 3).

\section{Enhanced association of SIR protein to $H M L$ in the gcn $5 \Delta$ cells}

Since SIR complex is prerequisite for the establishment and the maintenance of $H M L$ heterochromatin structure, we next examined if deletion of GCN5 altered the levels of $H M L$ bound Sir2 protein. Equal amounts of whole cell extracts from YXB4 (WT), the gcn5s strain and the sir3s strain were subjected to ChIP assay using the specific antibody of Sir2. Subsequently the DNA fragments of four regions spanning from silencer $\mathrm{E}$ to silencer I on $H M L$ locus were analyzed by PCR. As shown in Fig. 3A, Sir2-bounded fragments were observed on all four detected regions of NucS, Nuc8, Nuc7 and Nuc3 in the gcn5s cells, indicating that removal of GCN5 didn't affect the spreading of SIR complex on $H M L$. Furthermore, the amounts of all these four fragments were $\sim 2.5$ fold more in the gcn5 $\Delta$ cells than in WT (Fig. 3A, B), while the results of Western blots indicated that the cellular amounts of Sir2 were slightly increased in the gcn5 cells (Fig. 3C). These data suggested that the levels Sir2 bound at $H M L$ were increased due to the deletion of GCN5 from $S I R^{+}$cells. In addition, deletion of SIR3 impaired the association of Sir2 at the regions of NucS, Nuc8 and Nuc7 (Fig. 3A).

\section{Adverse effects of Gen5 and Sir3 on the HML circle topology}

To further evaluate the coordination of Gen5 and SIR complex on the regulation of $H M L$ structure, we compared the topology of $H M L$ circles isolated from the strains of $g c n 5 \Delta, \operatorname{sir} 3 \Delta$ and the double mutant gcn5s sir $\Delta$. Because the $F R T$ sequences were positioned differently in YXB4 and YXB2 (Fig. 1A), HML circles isolated from YXB4 (Fig. 4A, lanes 1-4) were smaller than those from YXB2 (Fig. 4A, lanes 5-8), which are $2.9 \mathrm{~kb}$ and $3.8 \mathrm{~kb}$, respectively (23). 
Deletion of GCN5 from YXB4 led to more negatively supercoiled $H M L$ circles (Fig. 4A, lane 2), confirming the earlier observations (Fig. 1B and 1F). Adversely, deletion of SIR3 resulted in total disruption of $H M L$ heterochromatin structure (Fig. 4A, lane 3), in agreement with previous work (23). Interestingly, the topology status of $H M L$ circles from the gcn5 sir $\Delta$ mutant was at an intermediate level between the status of the circles in the gcn5 $\Delta$ and the sir3 $\Delta$ cells (Fig 4A, lane 4). They were more condensed than the circles in sir3 $\Delta$ cells and were less condensed than the circles in gcn5 cells (Fig. 4B, right panel). Therefore, removal of GCN5 led to more compacted HML heterochromatin structure in both $S I R^{+}$cells and SIR- cells of YXB4.

Consistently, the $H M L$ heterochromatin circles became relaxed without Sir3 in YXB2 cells (Fig. 4A, lane 7). However, deletion of GCN5 from $S I R^{+}$cells and $S I R^{-}$cells of YXB2 didn't lead to detectable alterations of $H M L$ (Fig. 4A, lanes 6 and 8). Since the two recombination target sites for the Flp1 recombinase were inserted in YXB2 at positions flanking $H M L$ and including the $\mathrm{E}$ and I Silencers, instead of bracketing $H M L$ and excluding the silencers in YXB4 (Fig 1A), a reasonable explanation is that released $H M L$ circles with the silencers of YXB2 diminishes or interferes with the function of GCN5 on the heterochromatin structure through an unknown mechanism.

\section{Transcriptional silencing was promoted in the gcn $5 \Delta$ cells}

Because the removal of GCN5 let to a more compact heterochromatin structure and increased binding of SIR complex at $H M L$ locus, we assume that gene silencing at $H M L$ will be promoted in the $g c n 5 \Delta$ cells. To test this possibility, expression of the $U R A 3$ reporter gene replacing $H M L \alpha$ mating genes of $\alpha 1$ and $\alpha 2$ (Fig. 5A) (32) were determined by measuring cell survival rate in medium containing $0.1 \%$ of 5 -fluroorotic acid (FOA). As observed in previous work (33) and as shown in Fig. 5A, the gcn5s cells grew much slower than wild type (YXB61-1) in synthetic 
complete (SC) medium. Insertion of $U R A 3$ at $H M L$ increased the distance between Silencers $\mathrm{E}$ and I, consequently $U R A 3$ is not completely silenced and $\sim 18 \%$ cells of wild type grew on FOA plates (Fig. 5A and 5B), in agreement with our earlier work (8). Importantly, the amounts of survived cells was increased to about $25 \%$ ( $\sim 1.4$ fold) in the absence of GCN5, indicating that the URA3 gene was further silenced and the transcription of URA3 was reduced in the gcn5 cells. These data suggested that deletion of GCN5 enhanced gene silencing at $H M L$ locus.

\section{Reduced NER efficiency in the gcn $5 \Delta$ cells}

Gcn5 has been reported to play an important role in NER at a few transcribed genomic regions or non-transcribed genomic regions (11,34-36), its role in NER in heterochromatin, such as the $H M L$ locus has not been investigated. To examine whether NER efficiency at the heterochromatic $H M L$ is altered in the $g c n 5 \Delta$ cells, YXB4 cells were irradiated with $200 \mathrm{~J} / \mathrm{m}^{2}$ $\mathrm{UV}$, followed by additional growth in the dark for NER. Efficiency of NER was monitored using a PCR-based method developed previously (37). Equal amounts of genomic DNA were used for the individual PCR reactions, as indicated by the equal amounts of PCR products from amplifying a short $0.3 \mathrm{~kb} H M L$ fragment (Fig. 6A). When a $2.9 \mathrm{~kb}$ fragment was amplified, PCR efficiency reflected the NER efficiency because the existence of more DNA lesions on a longer DNA fragment blocks the Taq DNA polymerase. As a result, PCR products increased with the repairing time (Fig. $6 \mathrm{~A}$ ) and $\sim 65 \%$ of the $2.9 \mathrm{~kb}$ fragment was repaired $2 \mathrm{~h}$ after $\mathrm{UV}$ treatment (Fig. 6B and 6C). In contrast, deletion of GCN5 reduced the NER rate and $\sim 45 \%$ of the $H M L$ region was repaired $2 \mathrm{~h}$ after $\mathrm{UV}$ treatment (Fig 6B), 1.4-fold less than the wild type. These data indicated that Gen5 is involved in the NER process at the $H M L$ locus. Additionally, removal of SIR3 increased NER efficiency on the same $2.9 \mathrm{~kb}$ region of $H M L$ locus (Fig. 6C), consitent with 
bioRxiv preprint doi: https://doi.org/10.1101/2021.02.28.433071; this version posted February 28, 2021. The copyright holder for this preprint (which was not certified by peer review) is the author/funder. All rights reserved. No reuse allowed without permission.

a previous observation that NER efficiency at the repressed subtelomere region was enhanced in absence of SIR2 (38). 


\section{Discussion}

The data presented here revealed a role of the histone acetyltransferase Gen5 in the regulation of heterochromatin structure, DNA repair and gene silencing at the $H M L$ locus. We found that removal of Gen5 led to more compact heterochromatic $H M L$ and enhanced gene silencing. Furthermore, we revealed that Gen5 is involved in regulating the association of SIR complex with $H M L$ and in regulating NER efficiency of the heterochromatin. These findings shed light on a novel function of Gen5 on heterochromatin beyond its role on euchromatin as a transcription activator.

It is likely that the functions of Gen5 on the structure and the gene silencing of $H M L$ locus are related to its activity of acetylating nucleosomal histones. In $\mathrm{SIR}^{+}$cells of YXB4, Gen5 regulates the amounts of SIR bound with HML which is critical to the conformation of the heterochromatin $(5,8)$. Given that Gcn5 is a histone acetyltransferase and that SIR complex bind preferentially to unacetylated histones (2), it is possible that removal of Gen5 leads to further hypoacetylation of $H M L$ locus which increases the binding of SIR complex. Furthermore, the assumption suggests that there do exist certain levels of acetylated histones targeted by Gcn5 at $H M L$ locus to prevent heterochromatin hypercondensation, while most other histones at the heterochromatin are deacetylated by SIR complex $(13,39)$. Therefore, it is reasonable that SIR complex doesn't deacetylate all histones acetylated by Gcn5. Indeed, Gcn5 can acetylate histones at many different positions of histone $\mathrm{H} 3$ and $\mathrm{H} 4(13,16,40,41)$, and yet Sir2 specifically targets H3K9, H3K14 and H4K16 $(42,43)$. Importantly, the viewpoint is directly strengthened by the observation that deletion of Gen5 alters the heterochromatin structure of SIR ${ }^{-}$cells of YXB4 (Fig. 4A). Taken together, we presume that Gcn5-mediated histone acetylation regulates the conformation and gene silencing at the heterochromatic $H M L$ locus. 
Studies on NER at the repressed MFA2 promoter in budding yeast provides a significant view of how Gcn5 is involved in the NER process. Following UV irradiation, GG-NER (Global Genomic NER) complex Rad7-Rad16-mediated increase of Gcn5 occupancy at MFA2 promoter leads to elevated levels of histone acetylation of H3K9 and H3K14, which promotes an open chromatin structure at MFA2 and consequently increases NER efficiency $(11,20,44)$. Our primary observations indicate that only basal levels of Gen5 occupancy was observed at $H M L$ prior to UV treatment, whereas the occupancy was enhanced dramatically after UV irradiation (data not shown). Notably, we found that deletion of Gen5 impaired the NER efficiency at $H M L$ (Fig. 6B). However, the mechanism that Gen5 contributes to NER at heterochromatic $H M L$ is likely different from that at MFA2 promoter, since UV irradiation is unable to stimulate histone $\mathrm{H} 4$ or $\mathrm{H} 3$ acetylation at the repressed subtelomere due to the presence of Sir2 (38). Hence, how Gen5 is involved in NER of HML locus needs to be further investigated.

In summary, Gcn5 plays an important role in the structure maintenance, gene silencing and NER process at the $H M L$ heterochromatin. These findings suggest that the function of Gen5 at the heterochromatin correlates with its acetyltransferase activity targeting other histones instead of histone $\mathrm{H} 3 \mathrm{~K} 9$ and $\mathrm{H} 3 \mathrm{~K} 14$ at $H M L$ locus, which are deacetylated by the SIR complex. It is also possible that heterochromatin is more dynamic than we originally thought, there are always active competitions between the Gen5 histone acetyltransferases and the SIR deacetylase at the $H M L$ heterochromatin. Thus, it will be of considerable interest to profile a global map of histone acetylation at the heterochromatic $H M L$ in order to further evaluate the contribution of Gen5 to the heterochromatin. 


\section{Materials and Methods}

\section{Materials}

Pierce $^{\mathrm{TM}}$ high sensitivity streptavidin HRP and protein A agarose were purchased from Thermo Fisher Scientific ${ }^{\mathrm{TM}}$. Primary antibody of Sir2 was from Santa Cruz Biotechnology. Yeast synthetic complete medium were purchased from Clontech. Taq DNA polymerase was from Sigma Aldrich. Amersham hybond-N ${ }^{+}$nylon membrane was obtained from GE Healthcare Life Sciences. Oligonucleotides used in this work were synthesized by Sigma Aldrich. All other chemicals are molecular biology grade.

\section{Yeast strains}

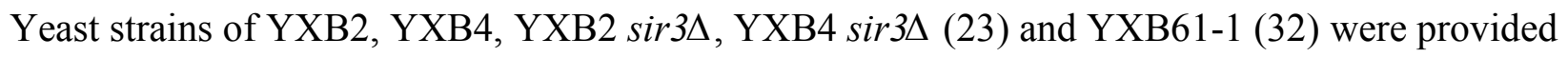
by Dr. Bi (University of Rochester). Single deletion mutants of Rad proteins and Gcn5 were

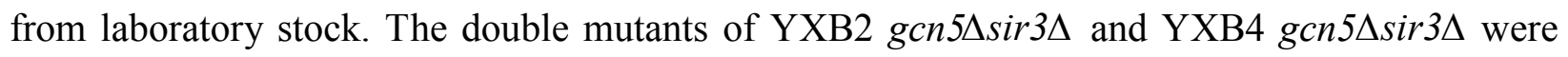
constructed in this work by removing GCN5 from the sir3 4 mutant using PCR-based gene deletion strategy $(45,46)$. All strains were confirmed by DNA sequencing. Absence of GCN5 were complemented by re-expressing GCN5 cloned into the expression vector YEp352 (47). GCN5 gene was amplified from genomic DNA of wild type (YXB4) using the primers HC01 and $\mathrm{HC} 02$ (Table S1).

\section{Topological analysis}

Topological structure of $H M L$ locus was analyzed as previously described (8). Yeast cells were grown to early log phase $\left(\mathrm{OD}_{600}=0.6\right)$ in YPR medium $(10 \mathrm{~g} / \mathrm{L}$ yeast extract, $20 \mathrm{~g} / \mathrm{L}$ peptone, $20 \mathrm{~g} / \mathrm{L}$ raffinose) at $30^{\circ} \mathrm{C}$. Galactose $(2 \%)$ was added to the culture that was further incubated for $2.5 \mathrm{~h}$ to induce the expression of Flp1 recombinase under control of GAL10 promoter. Genomic 
DNA was isolated using the glass bead method and was fractioned on $1.2 \%$ agarose containing $30 \mu \mathrm{g} / \mathrm{ml}$ of chloroquine, followed by transferred to the positively charged nylon membranes. $H M L$ circles were detected by Southern blotting using a $H M L$-specific probe (5'- biotinTCTTCTTGAGACAATTTGGCC-3') complementary to the $\alpha 1$ gene coding region (8). The 5'biotin labeled probe was incubated with membranes overnight at $45^{\circ} \mathrm{C}$. Membranes were washed twice with buffer I $(2 \times \mathrm{SSC}, 0.1 \% \mathrm{SDS})$ and buffer II $(0.2 \times \mathrm{SSC}, 0.1 \% \mathrm{SDS})$ before blocked using 3\% BSA prepared in PBST buffer. To detect $H M L$ circles, membranes were incubated with high sensitive streptavidin HRP overnight at $4{ }^{\circ} \mathrm{C}$, followed by detection with ECL reagents. The Gaussian center of topoisomer distributions was determined as described previously (8).

\section{Chromatin immunoprecipitation (ChIP)}

ChIP assays were performed as described in earlier work (8). Mid-log phase yeast cells were treated with $1 \%$ formaldehyde for $15 \mathrm{~min}$ at room temperature, pelleted, and washed twice with TBS (25 mM Tris, $\mathrm{pH} 7.5,137 \mathrm{mM} \mathrm{NaCl}, 2.7 \mathrm{mM} \mathrm{KCl}$ ). Crosslinked cells were suspended in lysis buffer (50 mM HEPES-KOH, pH 7.5, 140 mM NaCl, 1 mM EDTA, 1\% Triton X-100, 0.1\% sodium deoxycholate, $1 \mathrm{mM}$ PMSF, $1 \mathrm{mg} / \mathrm{ml}$ leupeptin, $1 \mathrm{mg} / \mathrm{ml}$ pepstatin A) and disrupted using glass beads, followed by sonication to yield DNA fragments with an average size of 300 bp. Protein concentration were measured by Bradford assay. Equal amounts of protein from each sample were incubated with specific antibody for Sir2 overnight at $4^{\circ} \mathrm{C}$. The immunocomplex was precipitated using Protein A sepharose beads. The beads were consecutively washed with the lysis buffer, wash buffer 1 (lysis buffer containing $500 \mathrm{mM} \mathrm{NaCl})$, wash buffer $2(10 \mathrm{mM}$ Tris-HCl, pH 8, $250 \mathrm{mM} \mathrm{LiCl,} \mathrm{0.5 \%} \mathrm{NP-40,} \mathrm{0.5 \%} \mathrm{sodium} \mathrm{deoxycholate,} 1 \mathrm{mM}$ EDTA) and TE buffer and then treated with RNase $\mathrm{A}$ in $\mathrm{TE}$ at $37^{\circ} \mathrm{C}$ for $30 \mathrm{~min}$. Chromatin was then eluted from the beads using elution buffer (1\% SDS, $\left.0.1 \mathrm{M} \mathrm{NaHCO}_{3}\right)$ and the cross-link reversed by 
incubation at $65^{\circ} \mathrm{C}$ overnight. DNA was then deproteinized by the addition of $4 \mu \mathrm{lof} 10 \mathrm{mg} / \mathrm{ml}$ proteinase $\mathrm{K}$ and incubation at $37^{\circ} \mathrm{C}$ for $2 \mathrm{~h}$. After phenol-chloroform extraction and ethanol precipitation, DNA was resuspended in $20 \mu \mathrm{l}$ of TE and was used as the templates for PCR assays. Total four regions of NucS, Nuc8, Nuc7 and Nuc3 were analyzed using $H M L$ specific primers. Respectively, NucS was amplified using the primers HC03 and HC04; Nuc8 was amplified using the primers HC05 and HC06; Nuc7 was amplified using the primers HC07 and HC08; Nuc3 was amplified using the primers HC09 and HC10. Nucleotide sequences of all these primers were listed in Table S1. PCR products were resolved on 1.5\% agarose gels.

\section{Western Blotting}

Total proteins were extracted from mid-log phase cells of WT and gcn5s. Protein concentration was measured by Bradford assay. $15 \mu \mathrm{g}$ of proteins were separated by $10 \%$ SDS-PAGE and were transferred to nitrocellulose membranes. Protein levels of Sir2 were detected using the protein-specific primary antibody.

\section{Analysis of NER efficiency on $H M L$ locus}

Cells of wild type and the gcn5 5 strain were grown to early-log phase in YPD medium at $30^{\circ} \mathrm{C}$. A part of cultures were harvested prior to UV treatment, the remained cultures were irradiated with $200 \mathrm{~J} / \mathrm{m}^{2} \mathrm{UV}$ light and were incubated additionally for different time ( $0 \mathrm{~h}, 0.5 \mathrm{~h}, 1 \mathrm{~h}$ and $\left.2 \mathrm{~h}\right)$ in the dark. Genomic DNA isolated from UV-treatment samples and control samples were used as PCR templates for analyzing NER efficiency at $H M L$ locus using a PCR-based technique developed previously (37). Rational of this technique is that DNA lesions on templates are able to block movement of PCR polymerase, resulting in decreased amplification. To monitor the process of DNA damage repair, a $2.9 \mathrm{~kb}$ fragment of $H M L$ was amplified from each sample 
using the primers $\mathrm{HC} 03$ and $\mathrm{HC} 10$ (Table S1). A $0.3 \mathrm{~kb}$ fragment of $H M L$ was amplified using the primers $\mathrm{HC} 03$ and $\mathrm{HC} 04$ (Table S1) to confirm equal amounts of genomic DNA was added into each PCR reaction. PCR products were detected on 1.0-1.5\% agarose gels and were quantified using Image $\mathrm{J}$ software. Efficiency of DNA damage repair at the $2.9 \mathrm{~kb}$ fragment was defined as the percentage of [(amounts of PCR products at each time points)/(amounts of PCR products of control)], in which the amounts of the $2.9 \mathrm{~kb}$ fragment were normalized using amounts of the $0.3 \mathrm{~kb}$ fragment.

\section{Analysis of gene silencing at $H M L$ locus}

Yeast cells of YXB61-1 and YXB61-1 gcn5s strains were grown to mid-log phase in YPD medium. To perform the spot assay, the cultures were first diluted to $\mathrm{OD}_{600}=0.005$, followed by making a 4-fold serial dilutions. $10 \mu \mathrm{l}$ of each diluted samples were spot out on the SC plates and the $\mathrm{SC}+0.1 \%$ FOA (5-fluoroorotic acid) plates. To calculate the cell survival rate, cultures were harvested by centrifuge and were resuspended in SC medium. The diluted cultures were spread on the SC plates and the SC+ $0.1 \%$ FOA plates and incubated at $30^{\circ} \mathrm{C}$ for $72 \mathrm{~h}$. Colony numbers were counted using Alphaimager 2000 software. Survival rate was defined as the percentage of [colony numbers on SC+ FOA plates]/ (colony numbers on SC plates)].

\section{Acknowledgments}

We thank Dr. Bi (University of Rochester) for providing us yeast strains. This work was supported by R01 ES017784 (to FG) from NIH. 


\section{References}

1. Bi, X. (2014) Heterochromatin structure: lessons from the budding yeast. IUBMB Life 66, 657-666

2. Rusche, L. N., Kirchmaier, A. L., and Rine, J. (2003) The establishment, inheritance, and function of silenced chromatin in Saccharomyces cerevisiae. Annu Rev Biochem 72, 481-516

3. Janssen, A., Colmenares, S. U., and Karpen, G. H. (2018) Heterochromatin: Guardian of the Genome. Annu Rev Cell Dev Biol 34, 265-288

4. Hahn, M., Dambacher, S., and Schotta, G. (2010) Heterochromatin dysregulation in human diseases. J Appl Physiol (1985) 109, 232-242

5. Kueng, S., Oppikofer, M., and Gasser, S. M. (2013) SIR proteins and the assembly of silent chromatin in budding yeast. Annu Rev Genet 47, 275-306

6. Yu, Q., Zhang, X., and Bi, X. (2011) Roles of chromatin remodeling factors in the formation and maintenance of heterochromatin structure. J Biol Chem 286, 14659-14669

7. Holmes, S. G., Rose, A. B., Steuerle, K., Saez, E., Sayegh, S., Lee, Y. M., and Broach, J. R. (1997) Hyperactivation of the silencing proteins, Sir2p and Sir3p, causes chromosome loss. Genetics $\mathbf{1 4 5}$, 605-614

8. Zhang, L., Chen, H., Bi, X., and Gong, F. (2013) Detection of an altered heterochromatin structure in the absence of the nucleotide excision repair protein Rad4 in Saccharomyces cerevisiae. Cell Cycle 12, 2435-2442

9. Rossl, A., Denoncourt, A., Lin, M. S., and Downey, M. (2019) A synthetic non-histone substrate to study substrate targeting by the Gen5 HAT and sirtuin HDACs. J Biol Chem 294, 6227-6239

10. Helmlinger, D., and Tora, L. (2017) Sharing the SAGA. Trends Biochem Sci 42, 850-861

11. Yu, Y., Teng, Y., Liu, H., Reed, S. H., and Waters, R. (2005) UV irradiation stimulates histone acetylation and chromatin remodeling at a repressed yeast locus. Proc Natl Acad Sci U S A 102, $8650-8655$

12. Burgess, R. J., and Zhang, Z. (2010) Roles for Gen5 in promoting nucleosome assembly and maintaining genome integrity. Cell Cycle 9, 2979-2985

13. Suka, N., Suka, Y., Carmen, A. A., Wu, J., and Grunstein, M. (2001) Highly specific antibodies determine histone acetylation site usage in yeast heterochromatin and euchromatin. Mol Cell 8, 473479

14. Downey, M., Johnson, J. R., Davey, N. E., Newton, B. W., Johnson, T. L., Galaang, S., Seller, C. A., Krogan, N., and Toczyski, D. P. (2015) Acetylome profiling reveals overlap in the regulation of diverse processes by sirtuins, gen5, and esa1. Mol Cell Proteomics 14, 162-176 
15. Lanza, A. M., Blazeck, J. J., Crook, N. C., and Alper, H. S. (2012) Linking yeast Gcn5p catalytic function and gene regulation using a quantitative, graded dominant mutant approach. PLoS One 7, e36193

16. Grant, P. A., Duggan, L., Cote, J., Roberts, S. M., Brownell, J. E., Candau, R., Ohba, R., OwenHughes, T., Allis, C. D., Winston, F., Berger, S. L., and Workman, J. L. (1997) Yeast Gen5 functions in two multisubunit complexes to acetylate nucleosomal histones: characterization of an Ada complex and the SAGA (Spt/Ada) complex. Genes Dev 11, 1640-1650

17. Grant, P. A., Eberharter, A., John, S., Cook, R. G., Turner, B. M., and Workman, J. L. (1999) Expanded lysine acetylation specificity of Gcn5 in native complexes. J Biol Chem 274, 5895-5900

18. Sun, J., Paduch, M., Kim, S. A., Kramer, R. M., Barrios, A. F., Lu, V., Luke, J., Usatyuk, S., Kossiakoff, A. A., and Tan, S. (2018) Structural basis for activation of SAGA histone acetyltransferase Gen5 by partner subunit Ada2. Proc Natl Acad Sci U S A 115, 10010-10015

19. Burgess, R. J., Zhou, H., Han, J., and Zhang, Z. (2010) A role for Gen5 in replication-coupled nucleosome assembly. Mol Cell 37, 469-480

20. Waters, R., van Eijk, P., and Reed, S. (2015) Histone modification and chromatin remodeling during NER. DNA Repair (Amst) 36, 105-113

21. Kuo, M. H., Zhou, J., Jambeck, P., Churchill, M. E., and Allis, C. D. (1998) Histone acetyltransferase activity of yeast Gcn5p is required for the activation of target genes in vivo. Genes Dev 12, 627-639

22. Jacobson, S., and Pillus, L. (2009) The SAGA subunit Ada2 functions in transcriptional silencing. Mol Cell Biol 29, 6033-6045

23. Bi, X., and Broach, J. R. (1997) DNA in transcriptionally silent chromatin assumes a distinct topology that is sensitive to cell cycle progression. Mol Cell Biol 17, 7077-7087

24. Hoeijmakers, J. H. (1993) Nucleotide excision repair I: from E. coli to yeast. Trends Genet 9, 173177

25. Jansen, L. E., Verhage, R. A., and Brouwer, J. (1998) Preferential binding of yeast Rad4.Rad23 complex to damaged DNA. J Biol Chem 273, 33111-33114

26. Oppikofer, M., Kueng, S., Martino, F., Soeroes, S., Hancock, S. M., Chin, J. W., Fischle, W., and Gasser, S. M. (2011) A dual role of H4K16 acetylation in the establishment of yeast silent chromatin. EMBO J 30, 2610-2621

27. Xu, E. Y., Bi, X., Holland, M. J., Gottschling, D. E., and Broach, J. R. (2005) Mutations in the nucleosome core enhance transcriptional silencing. Mol Cell Biol 25, 1846-1859 
28. Smith, C. M., Haimberger, Z. W., Johnson, C. O., Wolf, A. J., Gafken, P. R., Zhang, Z., Parthun, M. R., and Gottschling, D. E. (2002) Heritable chromatin structure: mapping "memory" in histones H3 and H4. Proc Natl Acad Sci U S A 99 Suppl 4, 16454-16461

29. Enomoto, S., and Berman, J. (1998) Chromatin assembly factor I contributes to the maintenance, but not the re-establishment, of silencing at the yeast silent mating loci. Genes Dev 12, 219-232

30. Huang, S., Zhou, H., Tarara, J., and Zhang, Z. (2007) A novel role for histone chaperones CAF-1 and Rtt106p in heterochromatin silencing. EMBO J 26, 2274-2283

31. Wu, M. Y., Lin, C. Y., Tseng, H. Y., Hsu, F. M., Chen, P. Y., and Kao, C. F. (2017) H2B ubiquitylation and the histone chaperone Asf1 cooperatively mediate the formation and maintenance of heterochromatin silencing. Nucleic Acids Res 45, 8225-8238

32. Bi, X., and Broach, J. R. (1999) UASrpg can function as a heterochromatin boundary element in yeast. Genes Dev 13, 1089-1101

33. Smith, E. R., Belote, J. M., Schiltz, R. L., Yang, X. J., Moore, P. A., Berger, S. L., Nakatani, Y., and Allis, C. D. (1998) Cloning of Drosophila GCN5: conserved features among metazoan GCN5 family members. Nucleic Acids Res 26, 2948-2954

34. Yu, S., Evans, K., van Eijk, P., Bennett, M., Webster, R. M., Leadbitter, M., Teng, Y., Waters, R., Jackson, S. P., and Reed, S. H. (2016) Global genome nucleotide excision repair is organized into domains that promote efficient DNA repair in chromatin. Genome Res 26, 1376-1387

35. Ferreiro, J. A., Powell, N. G., Karabetsou, N., Mellor, J., and Waters, R. (2006) Roles for Gcn5p and Ada2p in transcription and nucleotide excision repair at the Saccharomyces cerevisiae MET16 gene. Nucleic Acids Res 34, 976-985

36. Teng, Y., Yu, Y., and Waters, R. (2002) The Saccharomyces cerevisiae histone acetyltransferase Gen5 has a role in the photoreactivation and nucleotide excision repair of UV-induced cyclobutane pyrimidine dimers in the MFA2 gene. J Mol Biol 316, 489-499

37. Ayala-Torres, S., Chen, Y., Svoboda, T., Rosenblatt, J., and Van Houten, B. (2000) Analysis of gene-specific DNA damage and repair using quantitative polymerase chain reaction. Methods 22, $135-147$

38. Irizar, A., Yu, Y., Reed, S. H., Louis, E. J., and Waters, R. (2010) Silenced yeast chromatin is maintained by Sir2 in preference to permitting histone acetylations for efficient NER. Nucleic Acids Res 38, 4675-4686

39. Braunstein, M., Sobel, R. E., Allis, C. D., Turner, B. M., and Broach, J. R. (1996) Efficient transcriptional silencing in Saccharomyces cerevisiae requires a heterochromatin histone acetylation pattern. Mol Cell Biol 16, 4349-4356 
40. Zhang, W., Bone, J. R., Edmondson, D. G., Turner, B. M., and Roth, S. Y. (1998) Essential and redundant functions of histone acetylation revealed by mutation of target lysines and loss of the Gcn5p acetyltransferase. EMBO J 17, 3155-3167

41. Owen, D. J., Ornaghi, P., Yang, J. C., Lowe, N., Evans, P. R., Ballario, P., Neuhaus, D., Filetici, P., and Travers, A. A. (2000) The structural basis for the recognition of acetylated histone H4 by the bromodomain of histone acetyltransferase gen5p. EMBO J 19, 6141-6149

42. Imai, S., Armstrong, C. M., Kaeberlein, M., and Guarente, L. (2000) Transcriptional silencing and longevity protein Sir2 is an NAD-dependent histone deacetylase. Nature 403, 795-800

43. Tanny, J. C., and Moazed, D. (2001) Coupling of histone deacetylation to NAD breakdown by the yeast silencing protein Sir2: Evidence for acetyl transfer from substrate to an NAD breakdown product. Proc Natl Acad Sci U S A 98, 415-420

44. Yu, S., Teng, Y., Waters, R., and Reed, S. H. (2011) How chromatin is remodelled during DNA repair of UV-induced DNA damage in Saccharomyces cerevisiae. PLoS Genet 7, e1002124

45. Baudin, A., Ozier-Kalogeropoulos, O., Denouel, A., Lacroute, F., and Cullin, C. (1993) A simple and efficient method for direct gene deletion in Saccharomyces cerevisiae. Nucleic Acids Res 21, 33293330

46. Wach, A., Brachat, A., Pohlmann, R., and Philippsen, P. (1994) New heterologous modules for classical or PCR-based gene disruptions in Saccharomyces cerevisiae. Yeast 10, 1793-1808

47. Hill, J. E., Myers, A. M., Koerner, T. J., and Tzagoloff, A. (1986) Yeast/E. coli shuttle vectors with multiple unique restriction sites. Yeast 2, 163-167 


\section{Figure legends}

Figure 1. Analysis of $H M L$ circle topology in cells lacking Gen5, Rtt109, Asf1 and Caf1. (A) Schematic representation of $H M L$ locus. $H M L$ in the original yeast strain (top) and in the two artificial yeast strains YXB2 and YXB4 (bottom) are shown. White open arrows, $\alpha 1$ and $\alpha 2$ genes; open squares, $H M L$ silencers E and I; black open arrows, FRT (FLP1 recombination target) sites. A deleted $294 \mathrm{bp}$ fragment containing the divergent promoters of $\alpha 1$ and $\alpha 2$ is bracketed.

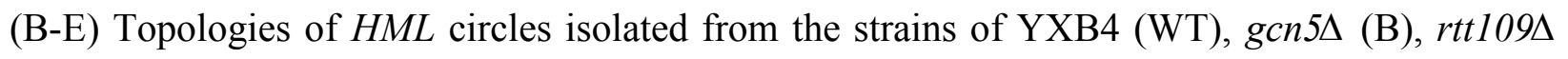

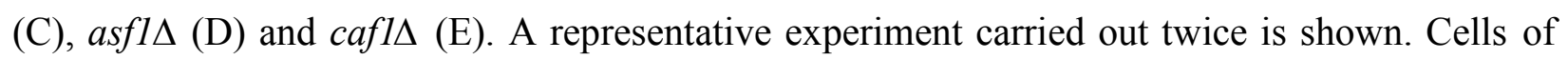
the indicated strains were grown to early log phase, followed by addition of galactose and additional incubation for $2.5 \mathrm{~h}$. To detect $H M L$ circles, $40 \mu \mathrm{g}$ of genomic DNA was fractioned by agarose gel electrophoresis in the presence of $30 \mu \mathrm{g} / \mathrm{ml}$ of chloroquine, subsequently transferred onto the membranes and subjected to Southern blotting as described in 'Materials and Methods'. $H M L$ topoisomers, nicked circles $(\mathrm{N})$ and the Gaussian center of topoisomer distribution (dots) are indicated. (F-I) Density profiles of the images B-E scanned by Image J software.

Figure 2. Topology analysis of $H M L$ circles in cells re-expressing Gen5. (A) Topologies of $H M L$ circles detected by Southern blotting. Expression of GCN5 was driven by its native promoter using a low copy expression vector. The empty vector or the vector taking GCN5 gene was introduced into YXB4. HML circles were formed by the induced recombination between the two FRT sites. To compare $H M L$ topology of the indicated strains, $40 \mu \mathrm{g}$ of genomic DNA isolated from the mid-log phase cells was subjected to Southern blotting as described in 'Materials and Methods'. HML topoisomers, nicked circles (N) and the Gaussian center of topoisomer distribution (dots) are indicated. A representative experiment carried out twice is shown. (B) 
Density profiles of the image in A scanned by Image J software. The density profiles of topoisomers are presented excluding the nicked circles.

Figure 3. Examination of the levels of Sir2 bound at $H M L$ locus. (A) Top panel, schematic positions of the four tested regions on $H M L$ of YXB4; Bottom panel, association of Sir2 with $H M L$ detected by ChIP. The DNA fragments bound with Sir2 were isolated using Sir2-specific antibody, subsequently the amounts of the purified DNA fragments of four individual regions (NucS, Nuc8, Nuc7, Nuc3) were analyzed by PCR as described in 'Materials and Methods'. A representative experiment carried out three times is shown. (B) Quantification of the data of Nuc7 in panel A. The levels of Sir2 bound at $H M L$ were determined by comparing the amounts of Sir2-associated DNA fragments (IP) with that of the total DNA fragments (input). The average \pm S.D. from three independent experiments is presented. (C) Sir2 expression in total cell extracts. $15 \mu \mathrm{g}$ of total protein from the two isogenic WTs (YXB4, BY4741) and the gcn5s strains was separated by $10 \%$ SDS-PAGE and subjected to Western blotting analysis. A representative experiment carried out three times is shown. Coomassie blue staining was used for the loading control.

Figure 4. Comparison of the topology of $H M L$ circles in the gcn5 5 and the gcn $5 \Delta$ sirs cells. (A) Detection of topology of $H M L$ circles by Southern blotting. $40 \mu \mathrm{g}$ of genomic DNA from the indicated strains was fractioned in agarose gels supplemented with $30 \mu \mathrm{g} / \mathrm{ml}$ chloroquine and was subjected to Southern Blotting analysis. Two sets of genomic DNA samples isolated from the strains under two wild type (YXB4, YXB2) backgrounds were used. A representative experiment carried out twice is shown. HML topoisomers, nicked circles $(\mathrm{N})$ and the Gaussian center of topoisomer distribution (dots) are indicated. (B) Density profiles of the image in A scanned by Image J software. 
Figure 5. Examination of gene silencing at $H M L$ locus in absence of Gen5. (A) Spot test assays of WT (YXB61-1) and the gcn5s cells. To test the gene silencing, a previous constructed strain YXB61-1 was used, in which the $H M L \alpha$ gene was replaced by the URA3 gene (top panel). Expression of the URA3 gene was monitored by spot test on $\mathrm{SC}$ plates and $\mathrm{SC}+0.1 \% \mathrm{FOA}$ plates as described in 'Materials and Methods'. A 4-fold serial dilution of the cultures of WT and the gcn $5 \Delta$ strain was made, $10 \mu 1$ of the diluted cultures was subsequently spotted out on the plates and incubated at $30^{\circ} \mathrm{C}$ for indicated time. A representative experiment carried out three times is shown. (B) Survival rate of WT and the $g c n 5 \Delta$ cells on the $\mathrm{SC}+0.1 \%$ FOA plates. To measure the survival rate of the cells on the FOA plates, the diluted cultures were spread on the plates and incubated at $30^{\circ} \mathrm{C}$ for $72 \mathrm{~h}$. Colony numbers were counted using Alphaimager 2000 software. Survival rate was calculated as described in 'Materials and Methods'. The average \pm S.D. from three independent experiments is presented.

Figure 6. Evaluation of NER efficiency at $H M L$ locus in the cells lacking Gen5. (A) Analysis of NER on a $2.9 \mathrm{~kb}$ fragment of $H M L$ in WT and the gcn5 cells. Cultures were harvested at the indicated time after $200 \mathrm{~J} / \mathrm{m}^{2}$ of UV treatment, followed by isolation of genomic DNA and PCR analysis as described in 'Material and Methods'. A $0.3 \mathrm{~kb}$ fragment of $H M L$ was amplified to monitor equal amounts of genomic DNA was used. (B) NER efficiency on the $2.9 \mathrm{~kb}$ fragment of $H M L$ in the gcn5s cells. The data in panel A was quantified and the NER efficiency was calculated as described in 'Materials and Methods'. (C) NER efficiency on the $2.9 \mathrm{~kb}$ fragment of $H M L$ in the sir3 $\Delta$ cells. For both $\mathrm{B}$ and $\mathrm{C}$, the average \pm S.D. from three independent experiments is presented. 


\section{Figure 1}

A

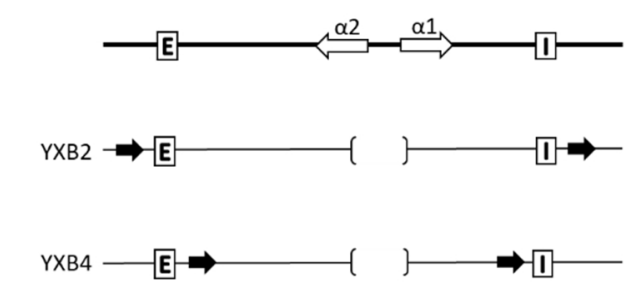

F

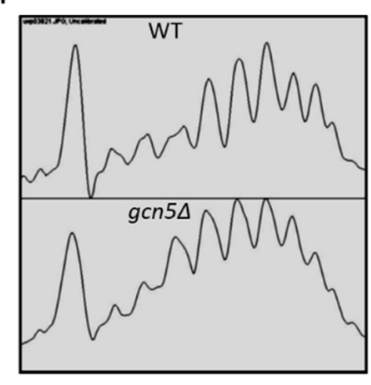

G

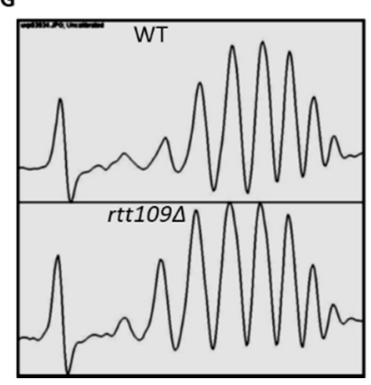

C

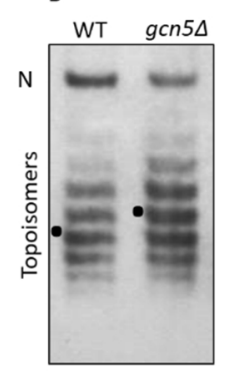

H

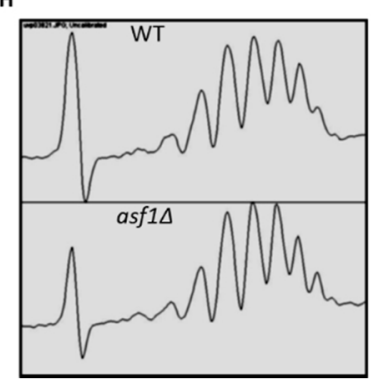

D

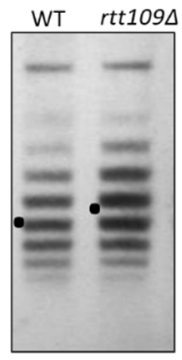

E

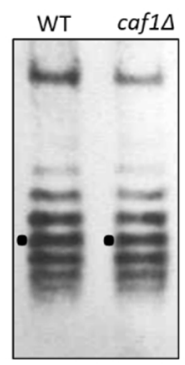

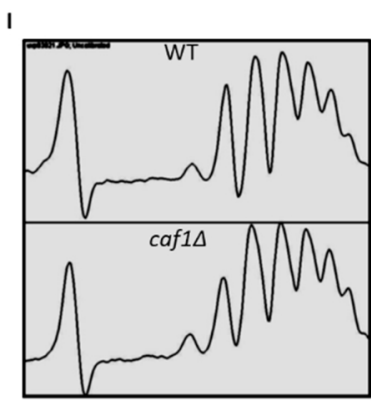


bioRxiv preprint doi: https://doi.org/10.1101/2021.02.28.433071; this version posted February 28, 2021. The copyright holder for this preprint (which was not certified by peer review) is the author/funder. All rights reserved. No reuse allowed without permission.

\section{Figure 2}

A

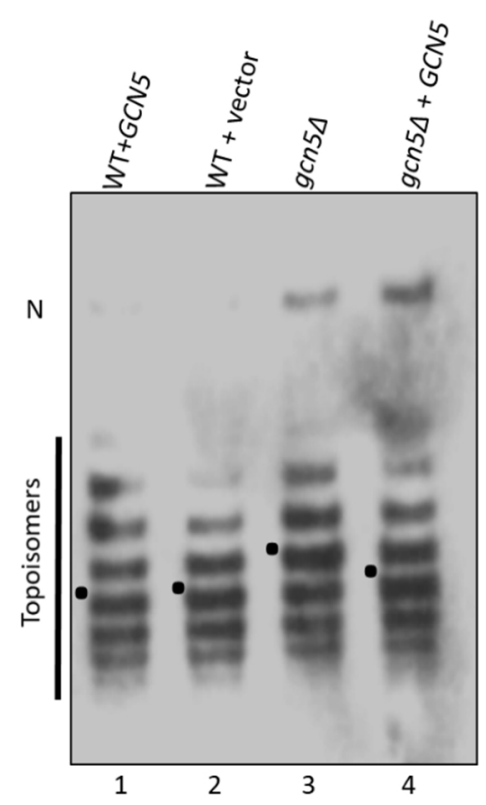

B

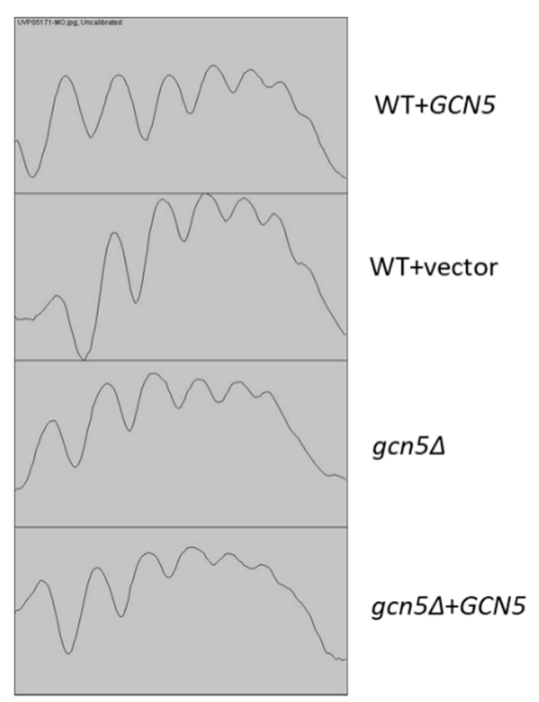


bioRxiv preprint doi: https://doi.org/10.1101/2021.02.28.433071; this version posted February 28, 2021. The copyright holder for this preprint (which was not certified by peer review) is the author/funder. All rights reserved. No reuse allowed without permission.

\section{Figure 3}

A

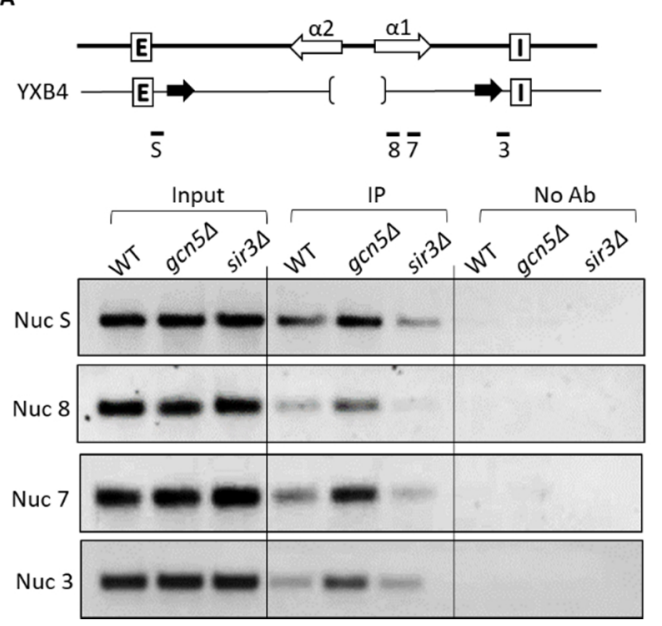

B

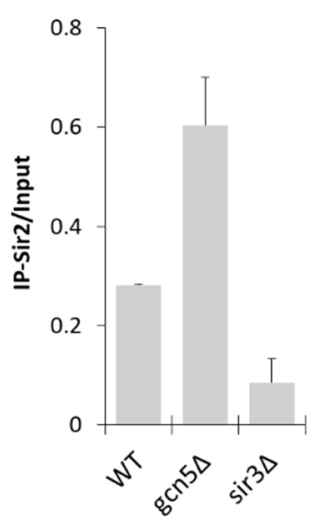

c

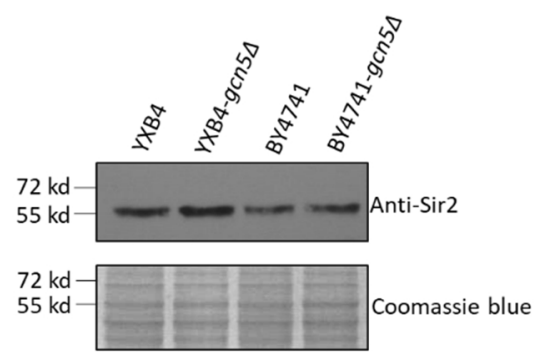




\section{Figure 4}

A

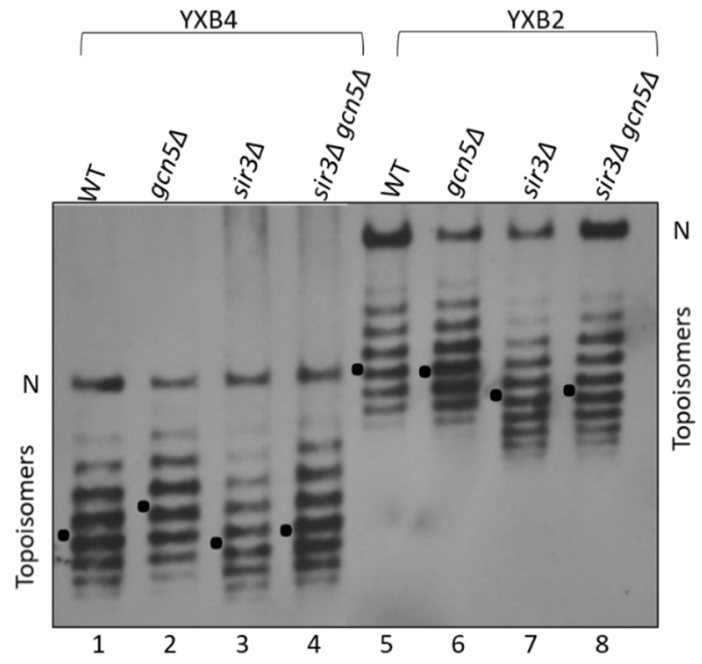

B

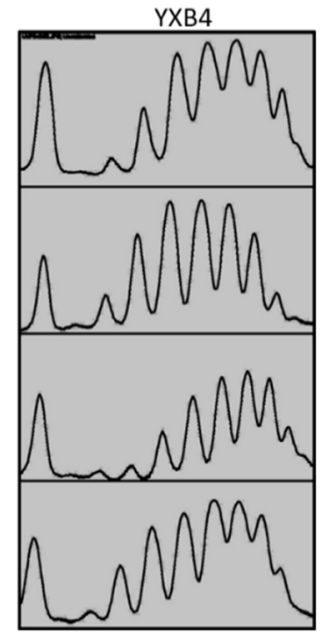

YXB2

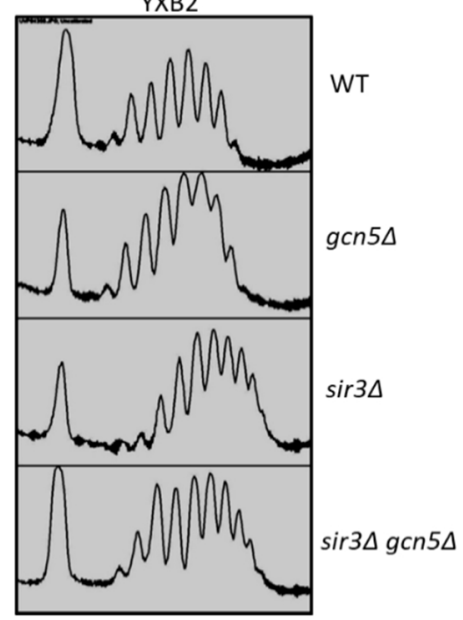


bioRxiv preprint doi: https://doi.org/10.1101/2021.02.28.433071; this version posted February 28, 2021. The copyright holder for this preprint (which was not certified by peer review) is the author/funder. All rights reserved. No reuse allowed without permission.

\section{Figure 5}

A

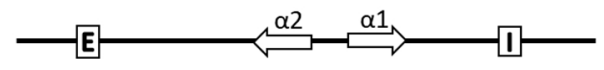

YXB61-1 E

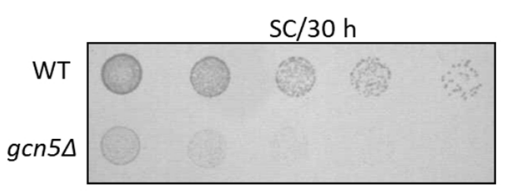

$\mathrm{SC} / 48 \mathrm{~h}$

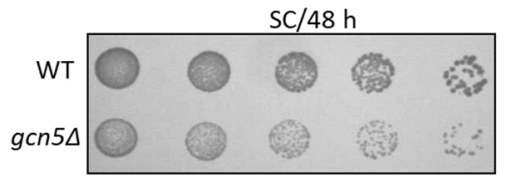

$\mathrm{SC}+0.1 \% \mathrm{FOA} / 54 \mathrm{~h}$

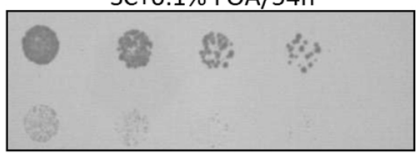

$\mathrm{SC}+0.1 \% \mathrm{FOA} / 72 \mathrm{~h}$

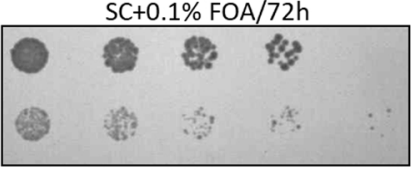

B

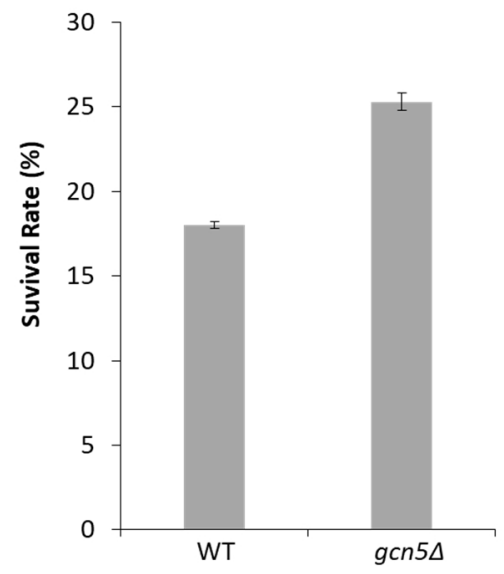




\section{Figure 6}

A

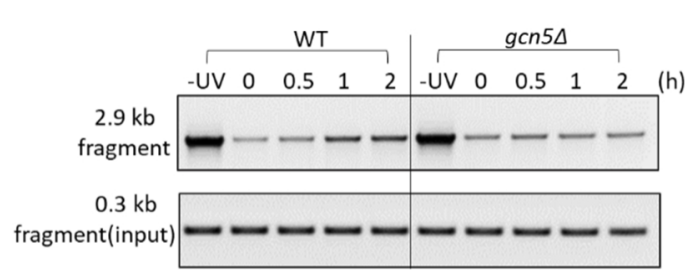

B

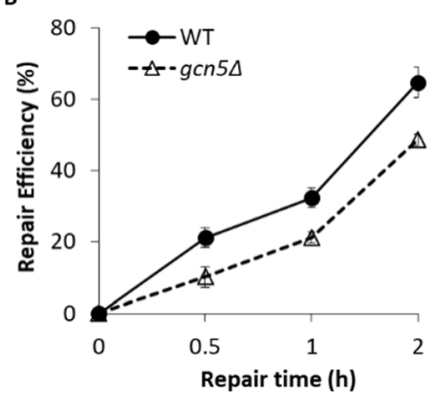

C

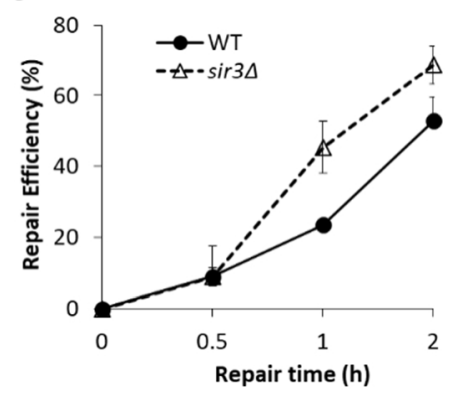

